\title{
An Integrodifference Model for Biological Invasions in a Periodically Fragmented Environment
}

\author{
Kohkichi KAWASAKI* and Nakako SHIGESADA \\ Faculty of Culture and Information Science \\ Doshisha University, Kyotanabe, 610-0321, Japan \\ * Tel: $+81-774-65-7677$ \\ Fax: $+81-774-65-7618$ \\ Email: kkawasak@mail.doshisha.ac.jp
}

Received June 24, 2006

Revised August 22, 2006

\begin{abstract}
An integrodifference model that describes the spread of invading species on a periodically fragmented environment is analyzed to derive an asymptotic speed of range expansion. We consider the case that the redistribution kernel is given by an exponentially damping function and the population growth is subject to a Ricker function in which the intrinsic growth rate is specified by a spatially periodic step-function. We first derive a condition for successful invasion of a small propagule, and then provide a mathematical formula for the rate of spread. Based on the speeds calculated from the formula for various combinations of parameter values, we discuss how the habitat fragmentation influences the invasion speed. The speeds are also compared with the corresponding speeds when the dispersal kernel is replaced by a Gaussian.
\end{abstract}

Key words: integrodifference model, periodically fragmented environment, traveling periodic wave, biological invasion

\section{Introduction}

When a species invades a new area, the success of invasion and its rate of spread are determined by interplay among dispersal, population growth, interactions with other species or environmental heterogeneity. Since the pioneer work of Skellam [38], various theoretical models have been developed to analyze spatio-temporal patterns of biological invasion. Earlier studies of invasion used reaction-diffusion equations on a homogeneous one-dimensional space, in which reproduction and movement are assumed to occur continuously and the movement is subject to random dispersal (Fisher [8], Skellam [38], Okubo [29], Bramson [4], Okubo and Levin $[30])$. Recently, newer models in various mathematical frameworks including integral kernel-based models (van den Bosch et al. [3], Mollison [26], Slatkin [39], Weinberger [42], Kot et al. [20], Metz et al. [25]), stratified diffusion model (Shigesada et al. [36], Shigesada and Kawasaki [34], [35]), cell-automata model (Shaw [33], Hastings [12], Ellner et al. [7], Kawasaki et al. [16]), and individual-based model (Higgins et al. [15]) have been developed in order to accommodate complex features in real ecosystems such as long distance dispersal, life-history of organisms, spatiotemporal heterogeneity, or demographic stochasticity (Hastings et al. [13]). Among them, the integrodifference model has been gaining growing attention for its ease in incorporating the life history of organisms with nonoverlapping generations and various types of dispersal kernel (Kot et al. [20], Veit and Lewis [41], Clark [6], 
Higgins and Richardson [14], Neubert and Caswell [27], Neubert et al. [28], Takasu et al. [40], Lewis and Pacala [22], Kot et al. [21], Lewis et al. [23]). However, few studies have examined the influence of spatial heterogeneity on the rate of spread in the framework of the integrodifference model.

Spatial heterogeneity in the invaded environment would have profound influence on the rate of spread as pointed out in a handful of empirical studies (Hastings et al. [13], Williamson and Harrison [45], With [46]). In the framework of reactiondiffusion equations, there have been a number of work that studied propagating waves in periodic media and proved the existence of the asymptotic front speed (Gartner and Freidlin [11], Freidlin [9], [10], Xin [47]). Recently Weinberger [43] presented a more general and strict mathematical formula for the asymptotic speed in periodically varying environments (see also Berestycki et al. [2], Berestycki and Hamel [1]). In the ecological context, Shigesada et al. [37] first presented a reactiondiffusion model for the spread of a single species in a patchy environment with periodic variations in diffusivity and growth rate and investigated how fragmentation of a favorable habitat could retard the rate of spread (see also Shigesada and Kawasaki [34], Kinezaki et al. [18], [17], Lutscher et al. [24]).

In this paper, we try to address the effect of spatial heterogeneity on invasions in the framework of the integrodiffence model. Our model involves an exponentially damping dispersal kernel and a spatially heterogeneous environment consisting of alternating favorable and unfavorable patches. Using computer simulations, we first demonstrate that when the trivial solution of the model is locally unstable, an initially localized population evolves to a propagating wave, in which the frontal pattern varies from year to year, but the average frontal speed tends to be constant. We further employ a heuristic method to obtain a mathematical formula for the average frontal speed. The speed calculated from the formula is illustrated as a function of the quality and the size of the unfavorable patch. Based on these results, we discuss how the environmental fragmentations influence the rate of spread of invasive species. Finally we examine how the speeds change when the dispersal kernel is replaced by a Gaussian kernel.

\section{Model}

Let us consider a single-species invasion in a one-dimensional environment, in which favorable and unfavorable habitats are alternately arranged in an infinite expanse of space. We assume that generations of the species are non-overlapping, so that individuals undergo reproduction and immediately die thereafter, as seen in an insect population with one generation each year. Then offspring redistribute according to a dispersal kernel, $k(x, y)$, which represents the probability density function for the location $x$ to which an individual at $y$ disperses. The redistributed offspring become adults within the same year, and reproduce their offspring. Thus if we denote by $N_{t}(x)$ the population density at location $x$ at the start of the $t$-th generation, the population density $N_{t+1}(x)$ at location $x$ and generation $t+1$ is given by summing up the population density over all location $y$, at which an 
individual is produced and then dispersed to location $x$ (Kot et al. [20]):

$$
N_{t+1}(x)=\int_{-\infty}^{\infty} k(x, y) F\left(N_{t}(y), y\right) N_{t}(y) d y \quad-\infty<x<\infty, \quad t=0,1,2, \ldots,
$$

where $F\left(N_{t}(y), y\right)$ is the per capita population growth rate at location $y$, which generally depends on both the population density and location $y$. Here we use the following growth function, which is modified from the Ricker equation [31]:

$$
F\left(N_{t}, x\right)=e^{r(x)-q N_{t}}
$$

where $q N_{t}$ represents the effect of intraspecific competition on the reproduction rate and $r(x)$ is the intrinsic growth rate at location $x$. In order to incorporate the heterogeneity of the patchy environment, we assume that $r(x)$ is given by a periodic step function as follows (see Fig. 1):

$$
r(x)=\left\{\begin{array}{lll}
r_{1} & \text { for } & n L-L_{1} \leq x<n L \\
r_{2} & \text { for } & n L \leq x<n L+L_{2}
\end{array}\right.
$$

where $L_{1}$ and $L_{2}$ respectively are the widths of the favorable and unfavorable habitats, and $L=L_{1}+L_{2}$. The intrinsic growth rates in the favorable and unfavorable habitats are assigned as $r_{1}$ and $r_{2}$, respectively. Note that $r_{1}$ should be positive, and $r_{2}$ is lower than $r_{1}$ and can be negative. When $r_{1}=r_{2}$, our model reduces to an integrodifference model in a homogeneous environment.

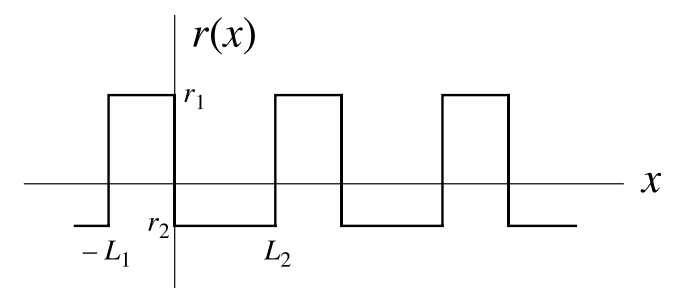

Fig. 1. A periodically fragmented environment. Favorable and unfavorable habitats with widths $L_{1}$ and $L_{2}$, and intrinsic growth rates $r_{1}$ and $r_{2}$, respectively, are arranged alternately in one-dimensional space.

As for the dispersal kernel, we adopt an exponentially damping function,

$$
k(x, y)=\frac{1}{2 d} \exp \left(-\frac{|x-y|}{d}\right)
$$

which can be derived under the assumption that dispersers move randomly and settle at a constant rate during sufficiently long dispersal period (Broadbent and Kendall [5], Williamson [44]). As the initial condition, we impose:

$$
N_{0}(x)=\left\{\begin{array}{lll}
\frac{N_{0}}{2 x_{0}} & \text { for } & |x| \leq x_{0} \\
0 & \text { for } & |x|>x_{0}
\end{array}\right.
$$




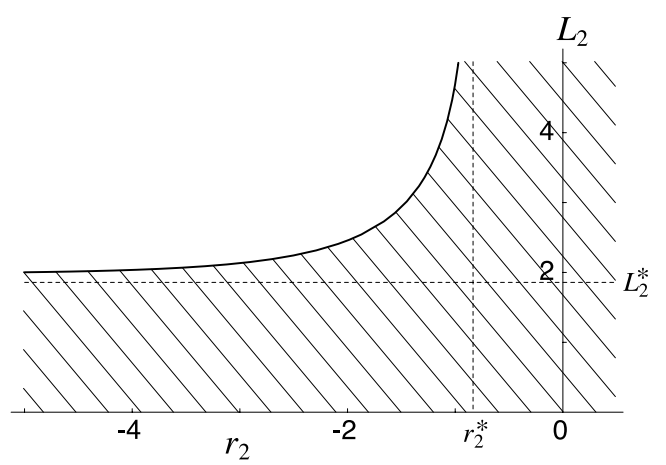

Fig. 2. Invasion condition in parameter space $\left(r_{2}, L_{2}\right)$ for $L_{1}=0.8, r_{1}=1$ and $d=1$. In the hatched region, invasion succeeds. $r_{2}^{*}=-0.855, L_{2}^{*}=1.9855$.

where $N_{0}$ is the number of organisms initially introduced in a small domain of $-x_{0} \leq x \leq x_{0}$. Now that an integrodifference model for a periodically fragmented environment is constructed by (1) with (2)-(5), we first examine the stability property of the trivial solution by analyzing the linearized equation of (1) about $N_{t}(x)=0$. Thus we obtain the following stability criteria:

The trivial solution is unstable,

$$
\begin{aligned}
& \text { if } e^{r_{2}} \geq 1 \text {, or } \\
& \text { if } e^{r_{2}}<1 \text { and } L_{1}>\frac{2 d}{\sqrt{e^{r_{1}}-1}} \operatorname{Tan}^{-1}\left\{\frac{\sqrt{1-e^{r_{2}}}}{\sqrt{e^{r_{1}}-1}} \tanh \left(\sqrt{1-e^{r_{2}}} \frac{L_{2}}{2 d}\right)\right\} \text {. }
\end{aligned}
$$

In other words, we may expect that a small number of introduced organisms will succeed in invasion if (6) holds; otherwise, the invasion will fail. In Fig. 2, the borderline for successful invasion is plotted in parameter space $\left(r_{2}, L_{2}\right)$ for $L_{1}=0.8$ and $r_{1}=1$. From this figure, we can see that if either $r_{2}$ is larger than a threshold, $r_{2}^{*}$, or $L_{2}$ is less than a threshold, $L_{2}^{*}$, invasion always succeeds. Furthermore, when $L_{2}$ is increased to infinity, $(6 \mathrm{~b})$ is reduced to

$$
L_{1}>\frac{2 d}{\sqrt{e^{r_{1}}-1}} \operatorname{Tan}^{-1} \frac{\sqrt{1-e^{r_{2}}}}{\sqrt{e^{r_{1}}-1}} .
$$

This means that if the width of the favorable patch $L_{1}$ is large enough to satisfy (7), invasion succeeds no matter how large the width of the unfavorable patch $L_{2}$ is. Moreover, even when the unfavorable patch is extremely hostile, i.e., $r_{2}=-\infty$ as well as $L_{2}=\infty$, invasion succeeds if

$$
L_{1}>\frac{2 d}{\sqrt{e^{r_{1}}-1}} \operatorname{Tan}^{-1} \frac{1}{\sqrt{e^{r_{1}}-1}},
$$

which coincides with the condition for persistence in a finite patchy domain with absorbing boundaries as previously derived by Kirk and Lewis [19]. 


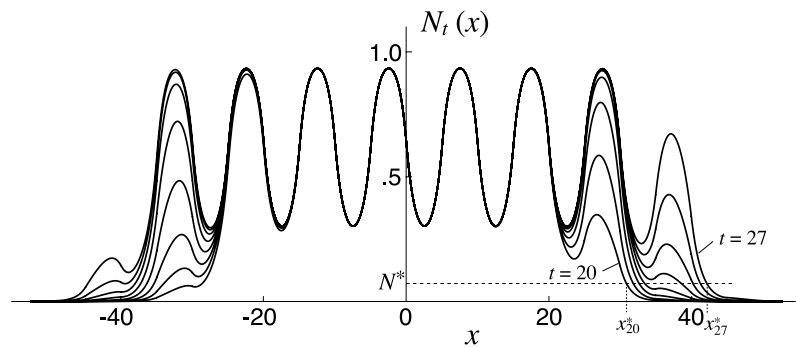

Fig. 3. A propagating wave that satisfies equation (1) with (2)-(5). Parameters are chosen as $r_{1}=1, r_{2}=0, L_{1}=5, L_{2}=5, q=1, d=1, x_{0}=1 / 2$, and $N_{0}=0.2$. $x_{t}^{*}$ indicates the location at which the population density of generation $t$ reaches a threshold, $N^{*}$.

Based on these results, we first carry out numerical simulations of the model for various parameter values. For the sake of simplicity, here we introduce the following variable transformation:

$$
N_{t}^{\prime}=q N_{t}, \quad x^{\prime}=\frac{x}{d}, \quad L_{1}^{\prime}=\frac{L_{1}}{d}, \quad L_{2}^{\prime}=\frac{L_{2}}{d} \quad \text { and } \quad L^{\prime}=\frac{L}{d} .
$$

Substituting (9) into (1)-(5) and omitting the primes, we have formally the same equation as (1)-(5) in which $q$ and $d$ are substituted by 1 . Therefore we subsequently put $q=d=1$ without loss of generality. From simulations, we found that when (6) does not hold, the solution will eventually becomes zero, so that invasion fails. On the other hand, when (6) is satisfied, the solution evolves into a propagating wave as shown in Fig. 3, where the population increases faster in the favorable habitats and slower in the unfavorable ones so that leading edge of the population continuously expands. It should be noted, however, the shape of the wave front varies with generation time, unlike the case of a traveling periodic wave as seen in the corresponding reaction-diffusion model, in which two successive waves are completely superimposed by shifting their mutual locations by one spatial period at every certain time interval (Shigesada et al. [37]). Nevertheless from a broader viewpoint, it looks like that the range expands at a roughly constant rate. To examine this point more precisely, here we define an instantaneous speed from time $t$ to time $t+1$ as $x_{t+1}^{*}-x_{t}^{*}$, where $x_{t}^{*}$ is the location of the front at which the population density $N_{t}(x)$ reaches a certain threshold of detection, $N^{*}$. By taking the average of the instantaneous speeds over time, we define the average frontal speed as,

$$
\tilde{c}=\lim _{t \rightarrow \infty} \frac{1}{t} \sum_{t^{\prime}=1}^{t}\left(x_{t^{\prime}}^{*}-x_{t^{\prime}-1}^{*}\right)=\lim _{t \rightarrow \infty} \frac{x_{t}^{*}}{t} .
$$

Fig. 4 shows the instantaneous speed, $x_{t^{\prime}}^{*}-x_{t^{\prime}-1}^{*}$, against $t$, which apparently varies from generation to generation. However, we found that the right hand side of (10) converges to a constant as $t$ goes to infinity. 


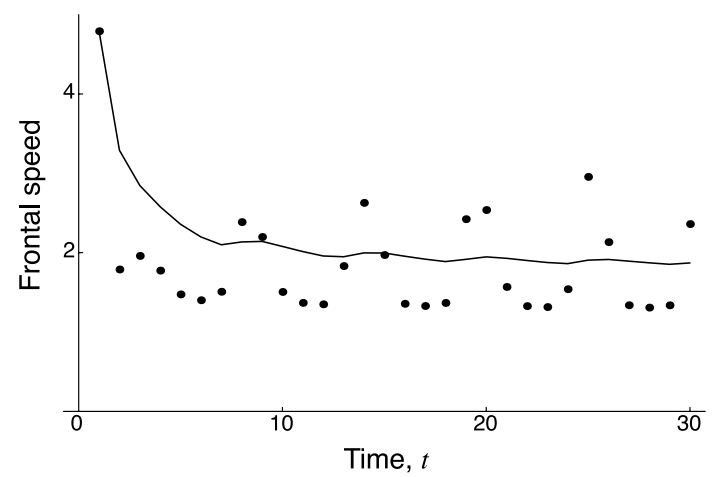

Fig. 4. Frontal speed as a function of generation time $t$. Closed circles are the instantaneous speed $x_{t+1}^{*}-x_{t}^{*}$, and the solid curve is the average of the instantaneous speed over $t$ generations. Parameters are the same as in Fig. 3.

\section{Mathematical formula for the average frontal speed-A heuristic method}

Here we present a heuristic method to derive the average frontal speed. Consider the case where (6) is satisfied so that the solution of (1) with (2)-(5) evolves to a propagating wave with average frontal speed $c$. We focus on the leading edge of the wave, where the population density is small. Then (1) can be approximated by the following linearized equation:

$$
n_{t+1}(x)=\int_{-\infty}^{\infty} k(x, y) R(y) n_{t}(y) d y
$$

where

$$
R(x)=\frac{\partial F}{\partial N_{t}}(0, x)=\left\{\begin{array}{lll}
e^{r_{1}} & \text { for } & n L-L_{1} \leq x<n L \\
e^{r_{2}} & \text { for } & n L \leq x<n L+L_{2}
\end{array}\right.
$$

In analogy to the reaction-diffusion model in a periodic patchy environment (Shigesada et al., 1984, Shigesada and Kawasaki, 1996), here we seek a solution of (11) in the following form:

$$
\begin{gathered}
n_{t}(x)=e^{-s(x-c t)} g(x), \\
g(x+L)=g(x),
\end{gathered}
$$

where $s$ is an auxiliary positive constant. For simplicity, we further confine ourselves to the case that $n_{t}(x)$ together with $d n_{t} / d x$ are continuous with respect to $x$. From (13a), we have a relation,

$$
n_{t+1}(x)=e^{s c} n_{t}(x)
$$


By substituting (14) into (11), we have

$$
e^{s c} n_{t}(x)=\int_{-\infty}^{\infty} k(x, y) R(y) n_{t}(y) d y
$$

We further substitute the dispersal kernel (4) into (15) to obtain:

$$
e^{s c} n_{t}(x)=\frac{1}{2} \int_{-\infty}^{x} e^{-x+y} R(y) n_{t}(y) d y+\frac{1}{2} \int_{x}^{\infty} e^{-y+x} R(y) n_{t}(y) d y .
$$

Differentiate the both sides of (16) with respect to $x$ twice. Then we have the following 2nd-order differential equation,

$$
n_{t}^{\prime \prime}(x)=\left(1-e^{-s c} R(x)\right) n_{t}(x)
$$

By substituting (13a) into (17) and rearranging the resultant equation, we get a differential equation for $g(x)$ as follows:

$$
g^{\prime \prime}(x)-2 s g^{\prime}(x)+\left\{s^{2}-\left(1-e^{-s c} R(x)\right)\right\} g(x)=0 .
$$

Since $R(x)$ is a periodic step function as defined in (12a), the general solution of (18) for $n L-L_{1}<x<n L+L_{2}$ is given by:

$$
g(x)=\left\{\begin{array}{lll}
g_{1}(x)=A_{1} e^{\left(s+q_{1}\right) x}+B_{1} e^{\left(s-q_{1}\right) x} & \text { for } & n L-L_{1} \leq x<n L \\
g_{2}(x)=A_{2} e^{\left(s+q_{2}\right) x}+B_{2} e^{\left(s-q_{2}\right) x} & \text { for } & n L \leq x<n L+L_{2}
\end{array}\right.
$$

where $A_{1}, A_{2}, B_{1}$, and $B_{2}$ are arbitrary constants and, $q_{1}$ and $q_{2}$ are given by

$$
q_{1}=\sqrt{1-e^{-s c} e^{r_{1}}}, \quad q_{2}=\sqrt{1-e^{-s c} e^{r_{2}}}
$$

Since we assumed that $g(x)$ is periodic with spatial period $L$ and that $n_{t}(x)$ and $d n_{t} / d x$ are continuous with respect to $x$, the following equations hold at the boundary of the favorable and unfavorable habitats:

$$
\begin{aligned}
& g_{1}(0)=g_{2}(0), \quad g_{1}\left(-L_{1}\right)=g_{2}\left(L_{2}\right), \\
& g_{1}^{\prime}(0)=g_{2}^{\prime}(0), \quad g_{1}^{\prime}\left(-L_{1}\right)=g_{2}^{\prime}\left(L_{2}\right) .
\end{aligned}
$$

Thus, substituting (19a) into (21), we have the following simultaneous equations:

$$
\left(\begin{array}{cccc}
1 & 1 & -1 & -1 \\
e^{-\left(s+q_{1}\right) L_{1}} & e^{-\left(s-q_{1}\right) L_{1}} & -e^{\left(s+q_{2}\right) L_{2}} & -e^{\left(s-q_{2}\right) L_{2}} \\
q_{1} & -q_{1} & -q_{2} & q_{2} \\
q_{1} e^{-\left(s+q_{1}\right) L_{1}} & -q_{1} e^{-\left(s-q_{1}\right) L_{1}} & -q_{2} e^{\left(s+q_{2}\right) L_{2}} & q_{2} e^{\left(s-q_{2}\right) L_{2}}
\end{array}\right)\left(\begin{array}{l}
A_{1} \\
B_{1} \\
A_{2} \\
B_{2}
\end{array}\right)=\left(\begin{array}{l}
0 \\
0 \\
0 \\
0
\end{array}\right)
$$




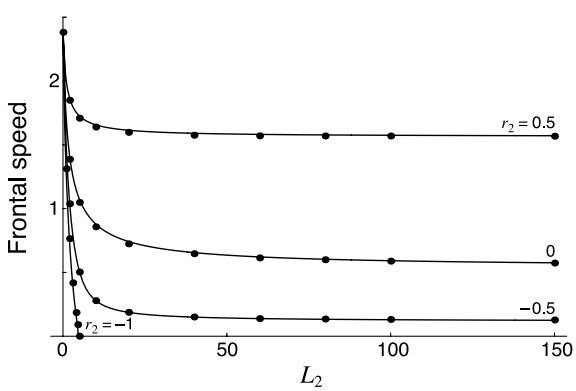

(a)

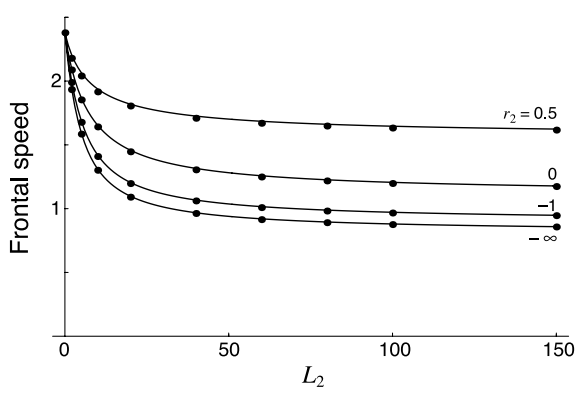

(b)

Fig. 5. Average frontal speeds calculated from mathematical formula (24) (solid curves) and from numerical simulations (closed circles) as functions of $L_{2}$. Other parameters are chosen as (a) $r_{2}=0.5,0,-0.5$ and $-1, L_{1}=0.8, r_{1}=1, q=1$ and $d=1$. (b) $r_{2}=0.5,0,-1$ and $\infty, L_{1}=5, r_{1}=1$ and $d=1$.

Since at least one of $A_{1}, A_{2}, B_{1}$ and $B_{2}$ should not be zero, by putting the determinant of the matrix of $(22)$ to be zero, we have a dispersion relation between $c$ and $s$ as follows:

$$
\cosh (s L)=\cosh \left(q_{1} L_{1}\right) \cosh \left(q_{2} L_{2}\right)+\frac{q_{1}^{2}+q_{2}^{2}}{2 q_{1} q_{2}} \sinh \left(q_{1} L_{1}\right) \sinh \left(q_{2} L_{2}\right) .
$$

If $c$ has a real root of (23) for a positive $s$, it should be a candidate of the average frontal speed. Following Weinberger [43] (see also Discussion section), we expect that the minimal value among the candidates of $c(s)$ gives the speed stably realized as a solution of (1):

$$
\bar{c}=\min _{0<s<\infty} c(s)
$$

To verify this, we calculate the speed from formula (24) for various combinations of $L_{1}, L_{2}, r_{1}$ and $r_{2}$. Fig. 5 (a) illustrates the average speed as a function of $L_{2}$ for varying values of $r_{2}$ with $L_{1}=0.8$ and $r_{1}=1$. The solid curves and the closed circles are theoretical and numerical results, respectively. Similarly, Fig. 5 (b) depicts the average speeds calculated for the same parameter sets as above except that $L_{1}$ is increased to 5 . On the other hand, Fig. 6 shows the average speed as a function of $r_{2}$ for varying $L_{2}$ with $L_{1}=5$ and $r_{1}=1$. Fig. 7 illustrates the average speed as a function of $r_{1}$ for varying values of $r_{2}$ with $L_{1}=5$ and $L_{2}=80$. As seen from all these figures, we can confirm that mathematical formula (24) perfectly fits with the average frontal speeds numerically calculated.

Finally it should be pointed out that when both $L_{1}$ and $L_{2}$ are sufficiently small, the dispersion relation (23) can be expressed in an explicit form as follows:

$$
c \approx \frac{1}{s}\left(\log \frac{e^{r_{1}} L_{1}+e^{r_{2}} L_{2}}{L_{1}+L_{2}}\right)+\log \frac{1}{1-s^{2}} \quad \text { for } \quad L_{1}, L_{2} \ll 1
$$




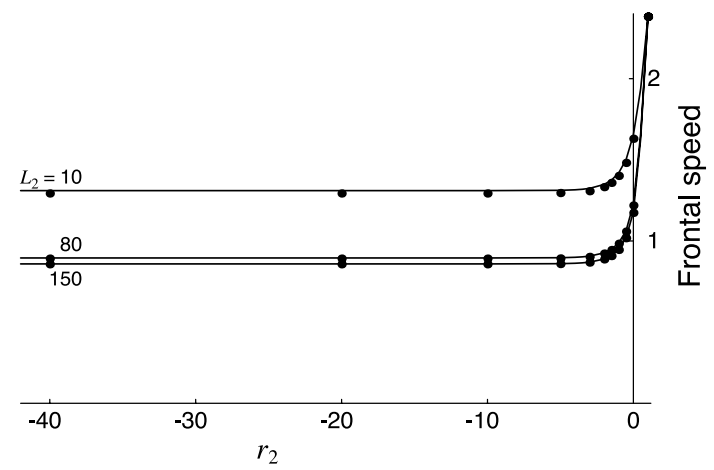

Fig. 6. Average frontal speeds calculated from mathematical formula (24) (solid curves) and from numerical simulations (closed circles) as functions of $r_{2}$. Other parameters are chosen as $L_{2}=10,80$ and $150, L_{1}=5, r_{1}=1, q=1$ and $d=1$.

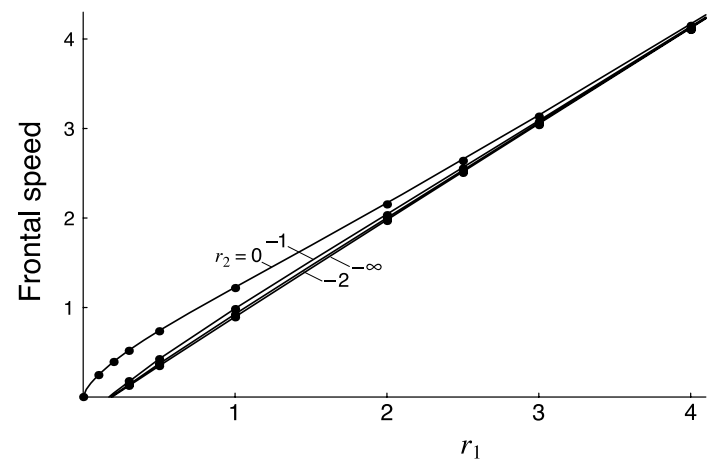

Fig. 7. Average frontal speeds calculated from mathematical formula (24) (solid curves) and from numerical simulations (closed circles) as functions of $r_{1}$. Other parameters are chosen as $r_{2}=0,-1,-2$ and $-\infty, L_{1}=5, L_{2}=80, q=1$ and $d=1$.

where approximation formulae, $\sinh z \approx z$ and $\cosh x \approx 1+z^{2} / 2$ for small $z$, are used. Thus if we define $e^{\bar{r}}$ as an arithmetic average of $e^{r(x)}$ :

$$
e^{\bar{r}}=\frac{e^{r_{1}} L_{1}+e^{r_{2}} L_{2}}{L_{1}+L_{2}}
$$

(25) appears to have the same form as the dispersion relation in a homogeneous environment (see Kot et al. [20]), in which the intrinsic growth rate is given by $\bar{r}$.

\section{Discussion}

There have been many elaborate works for range expansion of invading species using integrodiffrence equations (see Introduction section). However, they all dealt with a homogeneous environment. To our knowledge, the present work is the first 
to examine propagating waves in a periodic environment in the framework of integrodifference equation. We used a technique similar to that employed in the reaction-diffusion model by Shigesada et al. [37] and explicitly derived a mathematical formula for the average frontal speed. In the propagating waves that occurs in the integrodifference model, the frontal pattern changes from generation to generation so that it does not conform to the traveling periodic wave as seen in the reaction-diffusion model in a rigorous sense. This may be attributable to the fact that in the integrodifference model, time is restricted to be discrete. In fact, if $\left(L_{1}+L_{2}\right) / \bar{c}$ would happen to be an integer, the same frontal pattern should appear in every $\left(L_{1}+L_{2}\right) / \bar{c}$ generations. In any event, we could successfully derive a formula for the speed that exactly fit with the speed numerically obtained. Incidentally, Weinberger [43] previously obtained a mathematical formula for speed in general periodic media in the framework of recursions of the form $n_{t+1}=Q\left[n_{t}\right]$. The formula was derived under some hypotheses, among which $Q$ is assumed to be an order-preserving operator in the sense that if $u(x) \leq v(x)$ at every point, then $Q[u] \leq Q[v]$. Recently, Robbins and Lewis [32] rigorously analyzed an integrodifference model with an order-preserving operator, in which both the growth rate and the dispersal kernel vary with the habitat type. Although our formula is formally included in Weinberger's general formula, we numerically found that it holds without the order-preserving hypothesis. For example, in the cases of $r_{1}=2.5,3$ and 4 in Fig. 7, the order-preserving hypothesis is not satisfied, because the Ricker recruitment function, $N_{t} e^{r_{1}-N_{t}}$, in the favorable habitat is not monotone increasing with $N_{t}$ and has a hump to the left of the carrying capacity $r_{1}$, and the width of favorable habitat, $L_{1}=5$, is fairly large. Nevertheless, the solution of (1) advances in the form of (13a) at the leading edge, while far behind the front the spatio-temporal patterns exhibit periodic or chaotic oscillations. More rigorous mathematical justification of our heuristic formula, done in collaboration with $\mathrm{H}$. Weinberger, will be described elsewhere.

Hereafter, we discuss the characteristic features of the results as shown in Section 3 from an ecological point of view. Let us look at Fig. 5 (a). For each $r_{2}$, the average speed sharply decreases when $L_{2}$ increases from zero to around 10 , after which it reaches a plateau, if $r_{2}$ is larger than a certain number, i.e. -0.855 which corresponds to $r_{2}^{*}$ in Fig. 2. In contrast, when $r_{2}$ is smaller than $r_{2}^{*}$, the speed falls to zero if $L_{2}$ exceeds a certain value, which correspond to the value of $L_{2}$ on the invasion condition curve at $r_{2}$ in Fig. 2 (i.e., invasion condition (6) fails). On the other hand, when $L_{1}$ is increased to 5 (see Fig. 5 (b)), the speed quickly decreases at first and then tends to a positive asymptote, never falling to zero, no matter how large $L_{2}$ is. Conversely, Fig. 6 illustrates how the average speed is influenced by $r_{2}$ for various values of $L_{2}$. As $r_{2}$ decreases from 1 , the speed sharply decreases and soon reaches plateau. This means that when the intrinsic growth rate $r_{2}$ has a sufficiently large negative value, further decreases in $r_{2}$ has little effect on the average speed. Combining these results, we can say that even when both $L_{2}$ and $-r_{2}$ increase to infinity, the speed asymptotically approaches a positive constant, as long as $r_{1}$ and $L_{1}$ satisfy (8). Fig. 7 shows how the speeds 


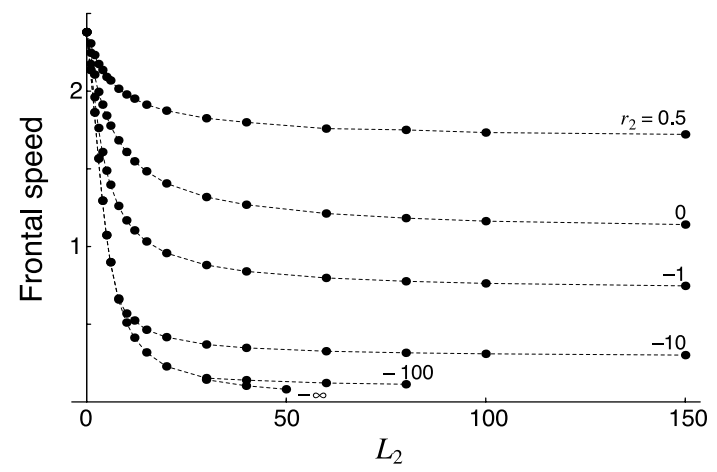

Fig. 8. Average frontal speeds in the integrodifference model with a Gaussian kernel as a function of $L_{2}$ (closed circles). Parameters chosen are $r_{2}=0.5,0,-1,-10,-100$ and $-\infty, L_{1}=5, r_{1}=1, q=1$ and $\sigma=1.68$. Because of the limit of computational accuracy, simulations for $r_{2}=-100$ and $-\infty$ are feasible only up to $L_{2}=80$ and 50 , respectively.

increases with increases in $r_{1}$ while other parameters are fixed constant. For each value of $r_{2}$, the average speed increases almost linearly with $r_{1}$ except when $r_{1}$ is small. This result is somewhat similar to the case in a homogeneous environment, in which the speed is approximately given by $c \approx d r_{1}$ for large values of $r_{1}$ (see Shigesada and Kawasaki [35]).

Although we have restricted ourselves to (4) for the dispersal kernel, we finally ask how the average speed is changed when the dispersal kernel (4) is replaced by a Gaussian:

$$
k(x, y)=\frac{1}{\sqrt{2 \pi \sigma^{2}}} \exp \left\{-\frac{(x-y)^{2}}{2 \sigma^{2}}\right\} .
$$

Unfortunately, we are not able to find an analytical formula for the average speed for the Gaussian kernel. Thus we carry out numerical simulations of (1) with (2), (3) and (27), and find a propagating wave similar to that for the case of the exponential kernel. Fig. 8 gives the speed corresponding to Fig. 5 (b), where all parameters except $\sigma$ are the same as in Fig. 5 (b), while $\sigma$ is chosen in such a way that the speed on the $y$ axis, namely, the speed in the homogenous environment, are the same between the two models. As in the case of the exponentially bounded kernel, the speed monotonically decreases with increases in $L_{2}$. However, this tendency is more prominent in the case of the Gaussian kernel: Most outstanding difference is that in Fig. 8, the curve for $r_{2}=-\infty$ comes closer to the $x$ axis as $L_{2}$ is increased (although numerical simulation is possible only up to $L_{2}=50$, owing to the limit of computational accuracy), while the speed in Fig. 5 (b) never reaches zero at the limit of $r_{2}=-\infty$ and $L_{2}=\infty$. These results imply that if two invasive species with an exponential kernel and a Gaussian kernel expand their range at the same speed in a homogeneous environment, fragmentation of environment prevents invasion more effectively for the species with the Gaussian kernel than that with the exponential 
kernel, and this tendency becomes more prominent as the unfavorable habitat is wider or harsher for survival.

Acknowledgments. We acknowledge Mr. Mitsugu Hisatomi for his valuable cooperation in the early stage of the present analyses. We are also very grateful to Dr. Hans Weinberger and Dr. Frithjof Lutscher for his important comments on our manuscript. This study was supported by the Grant-in-Aid for Scientific Research Fund from the Japan Ministry of Education, Science, Culture and Sports (no. 18570029).

\section{References}

[1] H. Berestycki and F. Hamel, Front propagation in periodic excitable media. Communications on Pure and Applied Mathematics, 55 (2002), 949-1032.

[2] H. Berestycki, N. Nadirashvili and F. Hamel, The speed of propagation for KPP type problems. I: Periodic framework. Journal of the European Mathematical Society, 7 (2005), $173-214$.

[ 3 ] F. van den Bosch, R. Hengeveld and J.A.J. Metz, Analyzing the velocity of animal range expansion. J. Biogeography, 19 (1992), 135-150.

[ 4 ] M. Bramson, Convergence of solutions of the Kolmogorov equation to traveling waves. Mem. Am. Math. Soc., 285 (1983), 1-190.

[ 5 ] S.R. Broadbent and D.G. Kendall, The random walk of Trichostrongylus retortaeformis. Biometrics, 9 (1953), 460-466

[6] J.S. Clark, Why trees migrate so fast: confronting theory with dispersal biology and the paleorecord. Am. Nat., 152 (1998), 204-224.

[ 7 ] S.P. Ellner, A. Sasaki, Y. Haraguchi and H. Matsuda, Speed of invasion in lattice population models: pair-edge approximation. J. Math. Biol., 36 (1998), 469-484.

[ 8 ] R.A. Fisher, The wave of advance of advantageous genes. Annals of Eugenics. (Lond.), 7 (1937), 355-369.

[ 9 ] M.I. Freidlin, On wavefront propagation in periodic media. Stochastic Analysis Applications, (ed. M. Pinsky) Advances in Probability and Related Topics, 7, M. Dekker, New-York, 1984, 147-166.

[10] M.I. Freidlin, Limit theorems for large deviations and reaction-diffusion equations. Ann. Probab., 13 (1985), 639-675.

[11] J. Gartner and M.I. Freidlin, On the propagation of concentration waves in periodic and random media. Soviet Math. Dokl., 20 (1979), 1282-1286.

[12] A. Hastings, Models of spatial spread: Is the theory complete? Ecology, 77 (1996), 1675-1679.

[13] A. Hastings, K. Cuddington, K.F. Davies, C.J. Dugaw, S. Elmendorf, A. Freestone, S. Harrison, M. Holland, J. Lambrinos, U. Malvadkar, B.A. Melbourne, K. Moore, C. Taylor and D. Thomson, The spatial spread of invasions: new developments in theory and evidence. Ecology Letters, 8 (2005), 91-101.

[14] S.I. Higgins and D.M. Richardson, Predicting plant migration rates in a changing world: The Role of Long-Distance Dispersal. Am. Nat., 153 (1999), 464-475.

[15] S.I. Higgins, D.M. Richardson and R.M. Cowling, Modeling invasive plant spread: The role of plant-environment interactions and model structure. Ecology, 7 (1996), 2043-2054.

[16] K. Kawasaki, F. Takasu, H. Caswell and N. Shigesada, How does stochasticity in colonization accelerate speeds of invasion in a cellular automaton model? Ecol. Res., 21 (2006), 334-345.

[17] N. Kinezaki, K. Kawasaki and N. Shigesada, Spatial dynamics of invasion in sinusoidally varying environments. Population Ecology, 48 (2006), 263-270.

[18] N. Kinezaki, K. Kawasaki, F. Takasu and N. Shigesada, Modeling biological invasions into periodically fragmented environments. Theor. Popul. Biology, 64 (2003), 291-302.

[19] R.W. Van Kirk and M.A. Lewis, Integrodifference models for persistence in fragmented habitats. Bull. Math. Biology, 59 (1997), 107-137. 
[20] M. Kot, M.A. Lewis and F. van den Driessche, Dispersal data and the spread of invading organisms. Ecology, 77 (1996), 2027-2042.

[21] M. Kot, J. Medlock, T. Reluga and D.B. Walton, Stochasticity, invasions, and branching random walks. Theor. Popul. Biology, 66 (2004), 175-184.

[22] M.A. Lewis and S. Pacala, Modeling and analysis of stochastic invasion processes. J. Math. Biol., 41 (2000), 387-429.

[23] M.A. Lewis, M.G. Neubert, H. Caswell, J.S. Clark and K. Shea, A guide to calculating discrete-time invasion rates from data. In Conceptual ecology and invasion biology: Reciprocal approaches to nature (eds. M.W. Cadotte, S.M. McMahon and T. Fukami), Springer, 2006, 169-192.

[24] F. Lutscher, M.A. Lewis and E. McCauley, Effects of heterogeneity on spread and persistence in rivers. Bull. Math. Biology, 68 (2006), 2129-2160.

[25] J.A.J. Metz, D. Mollison and F. van den Bosch, The dynamics of invasion waves. The Geometry of Ecological Interactions: Simplfying Spatial Complexity (eds. O. Dieckmann, R. Law and J.A.J. Metz), Cambridge University Press, 2000, 482-512.

[26] D. Mollison, Spatial contact model for ecological and epidemic spread. J. Rol. Stat. Soc. B, 39 (1977), 283-326

[27] M.G. Neubert and H. Caswell, Demography and dispersal: calculation and sensitivity analysis of invasion speed for structured populations. Ecology, 81 (2000),1613-1628.

[28] M.G. Neubert, M. Kot and M.A. Lewis, Invasion speeds in fluctuating environments. Proc. R. Soc. Lond. B, 267 (2000), 1603-1610.

[29] A. Okubo, Diffusion and Ecological Problems: Mathematical Models. Springer-Verlag, New York, 1980.

[30] A. Okubo and S.A. Levin, Diffusion and Ecological Problems: New Perspectives (second edition). Springer-Verlag, New York, 2001.

[31] W.E. Ricker, Stock and recruitment. J. Fisheries Research Board of Canada, 11 (1954), $559-623$

[32] T.C. Robbins and M.A. Lewis, Modeling population spread in heterogeneous environments using integrodifference equations. preprint, 2006.

[33] M.W. Shaw, Modeling stochastic processes in plant pathology. Annu. Rev. Phytopathol, 32 (1994), 523-544.

[34] N. Shigesada and K. Kawasaki, Biological Invasions; Theory and Practice. Oxford University Press, 1997.

[35] N. Shigesada and K. Kawasaki, Invasion and species range expansion: effects of long-distance dispersal. Dispersal Ecology (eds. J.M. Bullock, R.E. Kenward and R.S. Hails), Blackwell, Oxford, 2002, 350-373.

[36] N. Shigesada, K. Kawasaki and Y. Takeda, Modeling stratified diffusion in biological invasions. Am. Nat., 146 (1995), 229-251.

[37] N. Shigesada, K. Kawasaki and E. Teramoto, Traveling periodic waves in heterogeneous environments. Theor. Popul. Biology, 30 (1986), 143-160.

[38] J.G. Skellam, Random dispersal in theoretical populations. Biometrika, 38 (1951), 196-218.

[39] M. Slatkin, Gene flow and selection in a cline. Genetics, 75 (1973), 733-756.

[40] F. Takasu, N. Yamamoto, K. Kawasaki, T. Togashi, Y. Kishi and N. Shigesada, Modeling the expansion of an introduced tree disease. Biological Invasions, 2 (2000), 141-150.

[41] R.R. Veit and M.A. Lewis, Dispersal, population growth, and the Allee effect: dynamics of the house finch invasion of eastern North America. Am. Nat., 148 (1996), 255-274.

[42] H.F. Weinberger, Long-time behavior of a class of biological models. SIAM J. Math. Anal., 13 (1982), 353-396.

[43] H.F. Weinberger, On spreading speeds and traveling waves for growth and migration models in a periodic habitat. J. Math. Biol., 45 (2002), 511-548.

[44] E.J. Williamson, The distribution of larvae of randomly moving insect. Austral. J. Biol. Sci., 14 (1961), 598-604.

[45] E.J. Williamson and S. Harrison, The Biotic and abiotic limits to the spread of exotica revegetation species in oak woodland and serpentine habitats. Ecol. Appl., 12 (2002), 40-51.

[46] K.A. With, The landscape ecology of invasive spread. Cons. Biol., 16 (2002), 1192-1203.

[47] J. Xin, Front propagation in heterogeneous media. SIAM Review, 42 (2000), 161-230. 
